EPJ Web of Conferences 66, 03071 (2014)

DOI: $10.1051 /$ epjconf/ 20146603071

(C) Owned by the authors, published by EDP Sciences, 2014

\title{
Study of reactions induced by ${ }^{6} \mathrm{He}$ on ${ }^{9} \mathrm{Be}$
}

K.C.C. Pires ${ }^{1, a}$, R. Lichtenthäler ${ }^{2}$, A. M. Moro ${ }^{3}$, M. Rodriguez-Gallardo ${ }^{3}$, A. Lépine-Szily², V. Guimarães $^{2}$, P. N. Faria ${ }^{4}$, A. Barioni ${ }^{5}$, D.R. Mendes Junior ${ }^{4}$, M. Assunção ${ }^{6}$, M.C. Morais ${ }^{2}$, V. Morcelle $^{4, b}$, J.M.B. Shorto ${ }^{7}$, R. Pampa-Condori², V. Scarduelli², E. Leistenschneider ${ }^{2}$, L. M. Fonseca $^{2}$, V. Zagatto ${ }^{2}$, T. B. Nassar ${ }^{6}$, and J. C. Zamora ${ }^{8}$

${ }^{1}$ Universidade Tecnológica Federal do Paraná, Cornélio Procópio, Brazil

${ }^{2}$ Departamento de Física Nuclear, Instituto de Física, Universidade de São Paulo, Brazil

${ }^{3}$ Departmento de Física Atomica, Molecular y Nuclear, Universidad de Sevilla, Spain

${ }^{4}$ Instituto de Física, Universidade Federal Fluminense, Rio de Janeiro, Brazil

${ }^{5}$ Instituto de Física, Universidade Federal da Bahia, Brazil

${ }^{6}$ Departamento de Ciências Exatas e da Terra, Universidade Federal de São Paulo, Brazil

${ }^{7}$ Instituto de Pesquisa Energeticas e Nucleares, Comissão Nacional de Energia Nuclear, São Paulo, Brazil

${ }^{8}$ Technische Universität Darmstadt, Germany

\begin{abstract}
We present the results of experiments using a ${ }^{6} \mathrm{He}$ beam on a ${ }^{9} \mathrm{Be}$ target at energies $7-9$ times the Coulomb barrier. Angular distributions of the elastic, inelastic scattering (target breakup) and the $\alpha$-particle production in the ${ }^{6} \mathrm{He}+{ }^{9} \mathrm{Be}$ collision have been analysed. Total reaction cross sections were obtained from the elastic scattering analyses and a considerable enhancement has been observed by comparing to stable systems.
\end{abstract}

\section{Introduction and Experimental Setup}

Current experiments with reactions induced by exotic and weakly bound projectiles on heavy targets show interesting phenomena, such as the anomalously large values of the reaction cross sections [1$5]$, and long range absorption effects $[1,6]$. This work has been performed to investigate if the same holds for collisions with lighter targets, for which the Coulomb breakup is expected to be small.

The ${ }^{6} \mathrm{He}+{ }^{9} \mathrm{Be}$ measurements were performed using the RIBRAS facility [7] installed at the 8UD Pelletron Tandem accelerator Laboratory of the University of Sao Paulo. The RIBRAS system consists of two superconducting solenoids of $6.5 \mathrm{~T}$ maximum central field. The ${ }^{6} \mathrm{He}$ secondary beam, with intensity about $2.4 \times 10^{4} \mathrm{pps}$, was produced by the ${ }^{9} \mathrm{Be}\left({ }^{7} \mathrm{Li},{ }^{6} \mathrm{He}\right)$ reaction with a ${ }^{7} \mathrm{Li}$ primary beam of $300 \mathrm{nAe}$. The detection system consisted of four $\Delta \mathrm{E}-\mathrm{E}$ silicon telescopes, with 20 microns and 1000 microns of thickness, respectively, which allowed the identification of the ${ }^{6} \mathrm{He}$ particles from the ${ }^{7} \mathrm{Li}$ beam contaminant and light particles.

We have measured angular distributions for elastic scattering and $\alpha$-particle production from the interaction of ${ }^{6} \mathrm{He}$ on secondary targets of ${ }^{9} \mathrm{Be}\left(1.93 \mathrm{mg} / \mathrm{cm}^{2}\right)$ and ${ }^{197} \mathrm{Au}\left(2.95 \mathrm{mg} / \mathrm{cm}^{2}\right)$ at the energies

\footnotetext{
ae-mail: kellypires@utfpr.edu.br

bresent address: Universidade Federal de Itajubá, Campus Itabira, Minas Gerais, Brazil
} 
$\mathrm{E}_{\mathrm{lab}}=16.2 \mathrm{MeV}$ and $\mathrm{E}_{\mathrm{lab}}=21.3 \mathrm{MeV}$, between $15^{\circ}$ and $75^{\circ}$ in steps of $3^{\circ}$ in the laboratory system. The gold target was used for normalization and monitoring since the collision ${ }^{6} \mathrm{He}+{ }^{197} \mathrm{Au}$ at these energies are pure Rutherford. The Coulomb barrier for ${ }^{6} \mathrm{He}+{ }^{9} \mathrm{Be}$ system is $\mathrm{E}_{\mathrm{B}}=2.22 \mathrm{MeV}$ and for ${ }^{6} \mathrm{He}+{ }^{197} \mathrm{Au}$ system is $\mathrm{E}_{\mathrm{B}}=22.40 \mathrm{MeV}$. Typical biparametric $\Delta \mathrm{E}-\mathrm{E}$ spectra obtained in these experiments are shown in Figure 1.
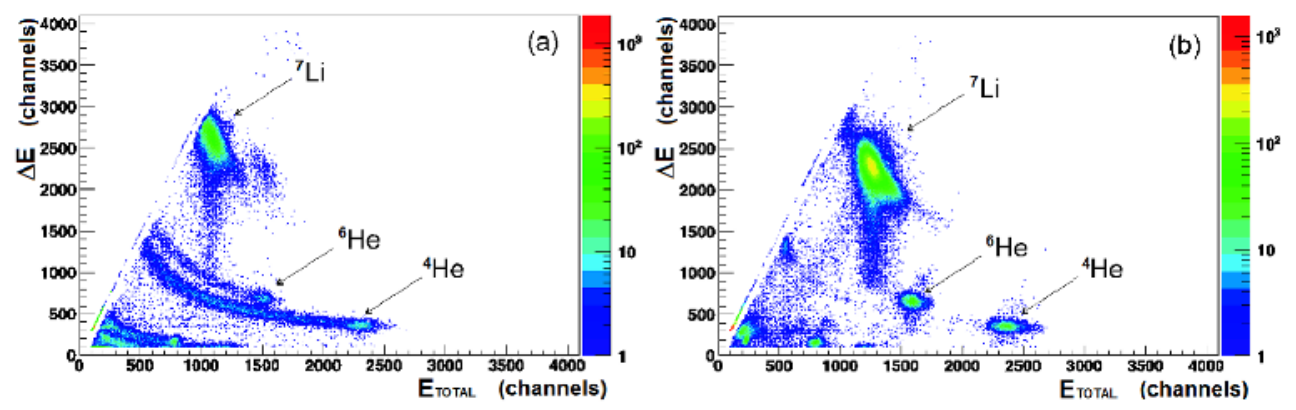

Figure 1. Biparametric spectra obained with ${ }^{6} \mathrm{He}$ beam on (a) ${ }^{9} \mathrm{Be}$, (b) ${ }^{197} \mathrm{Au}$ targets at $\theta_{\mathrm{lab}}=15^{\circ}$ at $\mathrm{E}_{\mathrm{lab}}=16.2 \mathrm{MeV}$. The ${ }^{7} \mathrm{Li}$ and ${ }^{4} \mathrm{He}$ elastic scattering peaks, at $\mathrm{E}_{\text {TOTAL }}$ around 1100 and 2350 channels respectively, come from the primary beam (contamination) and are indicated by arrows.

\section{Data Analysis and Results}

The ${ }^{6} \mathrm{He}+{ }^{9} \mathrm{Be}$ elastic scattering angular distributions have been obtained and were analysed by Coupled Channels (CC) calculations considering the ${ }^{9} \mathrm{Be}$ target excitation as well as by 3-body and 4-body Continuum-Discretization Coupled-Channels (CDCC) calculations including explicitly the breakup of the ${ }^{6} \mathrm{He}$ nucleus. The results of these calculations are shown in Figure 2 and more details are given in Ref. [6].
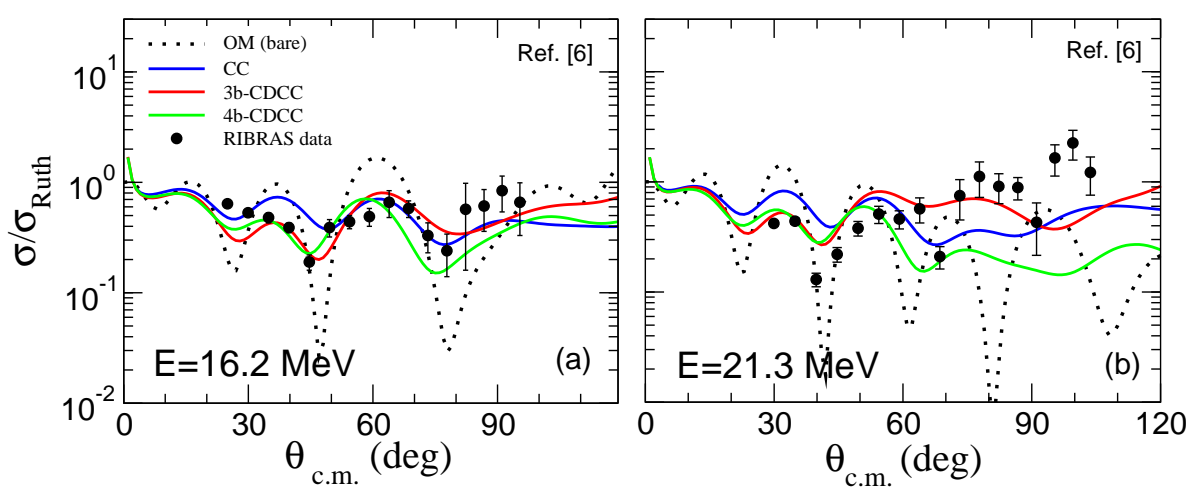

Figure 2. ${ }^{6} \mathrm{He}+{ }^{9} \mathrm{Be}$ elastic scattering angular distributions compared with several theoretical calculations at (a) $\mathrm{E}_{\mathrm{lab}}=16.2 \mathrm{MeV}$ and (b) $\mathrm{E}_{\mathrm{lab}}=21.3 \mathrm{MeV}$.

It is important to mention that the 3-body CDCC calculations presented in Figure 2 describe the ${ }^{6} \mathrm{He}$ states using an effective two body Hamiltonian $\left(2 \mathrm{n}+{ }^{4} \mathrm{He}\right)$ which reproduces the ${ }^{6} \mathrm{He}$ rms [8]. 
Nevertheless the 4-body calculations still present a reduced cross-section compared to the 3-body case, mainly at backward angles, which is not seen in the experimental data.

In addition to the interacting potentials, the elastic scattering calculations provide information of the total reaction cross section. For the analyses presented here (CC, 3b-CDCC, 4b-CDCC), the average total reaction cross sections are $1525(104) \mathrm{mb}$ and $1527(106) \mathrm{mb}$, respectively for $16.2 \mathrm{MeV}$ and $21.3 \mathrm{MeV}$ energies. These are relatively large values even when compared to the reaction cross sections for weakly bound stable projectiles, indicating that there is an enhancement of about $25 \%$ in the total reaction cross section for the ${ }^{6} \mathrm{He}+{ }^{9} \mathrm{Be}$ system with respect to stable systems.

Besides the elastic scattering events, a large $\alpha$-particle production is observed in the biparametric $\Delta \mathrm{E}-\mathrm{E}$ spectrum (see Figure 1) obtained with ${ }^{9} \mathrm{Be}$ target, which is not present in the spectrum with gold target [4]. It is to be noted that for ${ }^{6} \mathrm{He}+{ }^{197} \mathrm{Au}$ system, the energy is below the Coulomb barrier whereas for ${ }^{6} \mathrm{He}+{ }^{9} \mathrm{Be}$ case the energy is about 7-9 times the Coulomb barrier. It means that only a few reaction channels are open for the ${ }^{6} \mathrm{He}+{ }^{197} \mathrm{Au}$ case and Figure 1-(b) basically shows the beam content. The intensities of the contaminant beams have been obtained from a measurement performed at zero degrees using a fainted beam and are approximately $68.2 \%$ for the ${ }^{7} \mathrm{Li}, 7.7 \%$ for ${ }^{4} \mathrm{He}, 15.7 \%$ for ${ }^{6} \mathrm{He}$ and $8.4 \%$ for lighter particles $p, d, t$. Indeed, the large yield of $\alpha$-particles produced in the ${ }^{6} \mathrm{He}+{ }^{9} \mathrm{Be}$ collision indicates a large reaction cross section. We performed an estimation of the diferential $\alpha$ particle production cross section by integrating in energy the $\alpha$-counts and considering the total beam intensity, including the contaminants, and the cross sections range from 15 to $330 \mathrm{mb} / \mathrm{sr}$ in the angular range of the measurements.

Moreover, events related to the ${ }^{6} \mathrm{He}$ nucleus with energy smaller than the elastic scattering peak were observed in Figure 1-(a). These events are related to processes where the identity of the ${ }^{6} \mathrm{He}$ beam particles is preserved but not their energies and could correspond to the excitation of the ${ }^{9} \mathrm{Be}$ target since the ${ }^{6} \mathrm{He}$ nucleus has no bound excited states. As the ${ }^{9} \mathrm{Be}$ has no bound excited states either, we concluded that those events should correspond to ${ }^{9} \mathrm{Be}$ breakup. Another possible explanation to the inelastic events in the ${ }^{6} \mathrm{He}$ line would be the presence of multiple-scattering in the ${ }^{9} \mathrm{Be}$ target as the ${ }^{6} \mathrm{He}$ goes through it. The ${ }^{9} \mathrm{Be}$ target is rather thick, $10^{20}$ particles $/ \mathrm{cm}^{2}$ in comparison to the $10^{18}$ particles $/ \mathrm{cm}^{2}$ of the Gold target. However, inspection of the scattering probability, using the ${ }^{6} \mathrm{He}+{ }^{9} \mathrm{Be}$ Rutherford cross section for those scattering angles and the target thickness, gives an scattering probability of the order of $10^{-6}$ which would lead to $10^{-12}$ for double scattering which looks too small to affect our measurements.

A preliminary analyses for the angular distributions of the target breakup events shows cross sections ranging from $100-10 \mathrm{mb}$ in the angular range from $20-60$ degrees, which is a rather high cross section. Coupled Channel calculations for the ${ }^{9} \mathrm{Be}$ excitation of the two excited states $\left(5 / 2^{-}\right.$; $\mathrm{E}=2.43 \mathrm{MeV})$ and $\left(7 / 2^{-} ; \mathrm{E}=6.38 \mathrm{MeV}\right)$, assuming a collective model account for about only $1 / 5$ of the measured cross sections.

\section{Summary and Conclusions}

Experimental data for ${ }^{6} \mathrm{He}+{ }^{9} \mathrm{Be}$, measured at 16.2 and $21.3 \mathrm{MeV}$ using the RIBRAS facility, are presented. The angular distributions for the elastic scattering have been analysed in terms of the CC, 3b- and 4b-CDCC methods considering the projectile breakup [6].

A large yield of $\alpha$-particles has been observed in the spectra obtained with the ${ }^{9} \mathrm{Be}$ target, indicating a large reaction cross section. However, due to the presence of beam contaminants, it is not possible to conclude that those $\alpha$-particles are produced only in reactions induced by the ${ }^{6} \mathrm{He}$ beam. Probably, the beam contaminants, mainly ${ }^{7} \mathrm{Li}$ and $\alpha$-particles, are playing a role in the total $\alpha$-particle production cross section. 
Events of ${ }^{6} \mathrm{He}$ particles scattered with lower energy than the elastic peak have been measured and are related to the target breakup. Very high cross sections have been observed indicating that target breakup may give an important contribution to the total reaction cross section in the ${ }^{6} \mathrm{He}+{ }^{9} \mathrm{Be}$ collision.

\section{Acknowledgments}

The authors acknowledge the Fundação de Amparo à Pesquisa no Estado de São Paulo and Fundação Araucária for the financial support.

\section{References}

[1] P.N de Faria and et al, Phys. Rev. C 81, 044605 (2010).

[2] E.F. Aguilera and et al., Phys. Rev. C 83, 021601(R) (2011).

[3] K. Rusek and et al., Phys. Rev. C 72, 037603 (2005).

[4] P.N. de Faria and et al., Phys. Rev. C 82, 034602 (2010).

[5] A.M. Sánchez-Benítez and et al., Nuclear Physics A 803, 30 (2008).

[6] K.C.C. Pires and et al., Phys. Rev. C 83, 64603 (2011).

[7] R. Lichtenthäler and et al., Eur. Phys. Jour. A 25: 259-260, Suppl. 1 (2005).

[8] A.M. Moro and et al. Phys. Rev. C 75(6), 064607 (2007). 